\title{
Skin Biopsy is Predictive of Outcome in Experimental Sepsis by Multidrug- Resistant Pseudomonas aeruginosa
}

\author{
Vassiliki Tziortzioti ${ }^{1}$, Haritini Petropoulou ${ }^{2}$, Thomas Tsaganos ${ }^{1}$, Aikaterini Spyridaki ${ }^{1}$, \\ Maria Raftogiannis ${ }^{1}$, Evangelos J. Giamarellos-Bourboulis ${ }^{*}, 1$ and Nicolaos G. Stavrianeas ${ }^{2}$ \\ ${ }^{1} 4^{\text {th }}$ Department of Internal Medicine, and ${ }^{2} 2^{\text {nd }}$ Department of Dermatology and Venereology, University of Athens \\ Medical School, Greece
}

\begin{abstract}
To evaluate whether histological findings of skin in sepsis by Pseudomonas aeruginosa could be a predictive factor of progression to death, histological alterations after challenge by one multidrug-resistant isolate were studied in 24 rabbits. Acute pyelonephritis was induced after ligation of the right ureter and injection of $10^{8} \mathrm{CFU}$ per kg of body weight into the renal pelvis. Biopsy samples of skin were taken on necropsy. Mean survival of animals after bacterial challenge was 5.23 days. Main histological findings of skin were inflammation and swelling of dermis; thickening of endothelium; presence of thrombi in vessels; necrobiotic changes of the hair follicles. Serum TNF $\alpha$ was negatively correlated to histology of dermis and follicles. Positive correlation was found between survival and swelling of dermis. It is concluded that prolongation of survival was accompanied by intense edema of the dermis. A punch skin biopsy might be a predictive factor of sepsis outcome.
\end{abstract}

Keywords: Inflammation, dermis, Pseudomonas aeruginosa.

\section{INTRODUCTION}

Pseudomonas aeruginosa is a common pathogen that is involved in nosocomial sepsis and is often characterized nowadays by multidrug-resistance [1]. It is associated with a poor prognosis despite continuous improvements in intensive care medicine and antibiotic therapy. Alterations of skin and its vasculature are important in the phenomenon of sepsis. It has been shown previously [2] that sepsis following multidrug-resistant $P$. aeruginosa infection is correlated with prolonged survival and greater inflammatory response in the dermis compared to infection by susceptible $P$. aeruginosa. As these cutaneous alterations could predict time interval to death, a single punch skin biopsy might be of value.

The present study focused on the presence of changes of epidermis, dermis, subcutaneous tissue, cutaneous vasculature and hair follicles following challenge by multidrugresistant $P$. aeruginosa. Principal aim was to assess these cutaneous alterations in relation to survival of experimental animals as well as to define whether a punch skin biopsy might be a significant indicator of progression to death.

\section{ANIMALS AND METHODOLOGY}

\section{Animals}

A total of 24 white New Zealand male rabbits of a mean ( \pm SD) weight of $3.29 \pm 0.41 \mathrm{kgr}$ were included in the study. The study received a permit from the Veterinary Directorate of the Perfecture of Athens, according to Greek legislation, in conformity to the Council Directive of the European Union. Animals were housed individually in metal cages and

*Address correspondence to this author at the $4^{\text {th }}$ Department of Internal Medicine, 'Attikon' University General Hospital, 1 Rimini Str., Athens GR124 62, Greece; Tel: +30 2105831 994; Fax: +30 2105326 446;

E-mail: giamarel@ath.forthnet.gr or giamarel@yahoo.com had access to tap water and standard balanced rabbit chow ad libitum. Room temperature ranged between 18 and $22^{\circ} \mathrm{C}$, relative humidity between 55 and $65 \%$ and the light-dark cycle was 12 hours (lights on at 6 am and off at $6 \mathrm{pm}$ ). Rabbits were selected for the study because of the easiness to induce experimental sepsis resembling the stages of human sepsis [3].

\section{Bacterial Isolate}

One multidrug-resistant isolate of $P$. aeruginosa derived from the blood of a patient with severe nosocomial sepsis was applied. Multidrug-resistance of the test isolate was assessed after estimation of minimal inhibitory concentrations (MICs) of ticarcillin-clavulanate, piperacillin, ceftazidime, imipenem, meropenem, ciprofloxacin and amikacin by a microdilution technique according to clinical and laboratory standards institute [4]. MICs of the above antimicrobials were $>256 / 2,>128,16,16,16,>128$ and $>256 \mu \mathrm{g} / \mathrm{ml}$ respectively.

\section{Study Design}

Acute pyelonephritis was induced as described previously $[3,5,6]$. Animals were initially sedated by the intramuscular injection of $25 \mathrm{mg} / \mathrm{kg}$ of ketamine and $5 \mathrm{mg}$ of $\mathrm{xy}-$ lazine per $\mathrm{kg}$. Anesthesia was maintained by the intramuscular administration of $15 \mathrm{mg} / \mathrm{kg}$ of xylazine at $30-\mathrm{min}$ time intervals. The peritoneal cavity was entered through an upper midline abdominal incision and the intestines were displaced to the left. The right ureter was recognized and ligated with a 3.0 suture just below the pelvis. A total of $10^{8} \mathrm{CFU}$ of the $P$. aeruginosa isolate per $\mathrm{kg}$ in a volume of $0.1 \mathrm{ml}$ was injected into the renal pelvis, proximal to the suture, with a 26-gauge needle. The peritoneal cavity and the abdominal wall were then closed by layers. 
Survival of animals was recorded each $12 \mathrm{~h}$ for a total period of follow-up of 21 days. Blood samples were collected after venipuncture of the right ear vein at 24 and 48 hours for estimation of tumour necrosis factor-alpha (TNF $\alpha$ ). Necropsy was performed after death; biopsy samples of 3 $\mathrm{cm}^{3}$ of skin and its appendages were taken by simple incision from the dorsal surface of the left femoral area by all animals. Animals that remained alive after 21 days of follow-up were killed by the intravenous administration of sodium thiopental. Cutaneous biopsies were taken on necropsy, as described above.

\section{Histology}

Tissue samples were fixed in neutral buffered formalin, embedded in paraffin wax, sectioned and stained with hematoxylin and eosin. Tissue sections were examined by two separate expert pathologists. Each tissue section was scored by a qualitative climax ranging between 0 and 3: (absent: 0 , mild: 1 , moderate: 2 , intense: 3 ) according to the experience of the pathologists concerning the following elements of epidermis, dermis and subcutaneous tissue.

Epidermis: necrobiotic elements and presence of necrosis and/or erosion; dermis: infiltration by neutrophils and edema; vessels of dermis or subcutaneous tissue: eosinophilic degeneration, thickening of endothelium, extravasation of red cells, presence of thrombi in vessels and inflammatory cells; hair follicles of dermis or subcutaneous tissue: necrobiotic changes, necrosis, acantholytic cells within the follicular wall and dyskeratotic cells.

\section{Estimation of Serum Levels of TNFa}

Blood samples were centrifuged and serum was kept refrigerated at $-70^{\circ} \mathrm{C}$ until assayed. TNF $\alpha$ was measured by a bioassay on L929 fibrosarcoma cell line, as already described [6]. Briefly, confluent cells were thoroughly washed with Hank's solution and harvested with $0.25 \%$ thrypsin/0.02\% EDTA (Biochrom AG, Berlin, Germany). Cells were centrifuged, re-suspended in RMPI 1640 supplemented with $10 \%$ Fetal Bovine Serum and $2 \mathrm{mM}$ of glutamine (Biochrom AG) and distributed into a 96-well cell culture plate at a density of $1 \times 10^{5}$ cells/well. The final volume of fluid into each well was $0.05 \mathrm{ml}$. After incubation for $2-3$ hours at $37^{0} \mathrm{C}$ with $5 \% \mathrm{CO}_{2}, 0.06 \mathrm{ml}$ of serum or of standard dilutions of known concentrations of human TNFa (Sigma, range 5.75$375.00 \mathrm{pg} / \mathrm{ml}$ ) were added into each well followed by 0.05 $\mathrm{ml}$ of a $0.3 \mathrm{mg} / \mathrm{ml}$ dilution of cycloheximide (Sigma). Incubation continued over-night; then the supernatant of each well was discarded by aspiration and $0.1 \mathrm{ml}$ of a $0.5 \mathrm{mg} / \mathrm{ml}$ methylene blue solution in methanol $99 \%$ was added. After ten minutes, the dye was removed and wells were thoroughly washed three times with $0.9 \%$ sodium chloride. Wells were left to dry and remnants of the dye in each well became soluble by the addition of $0.1 \mathrm{ml}$ of $50 \%$ glacial acetic acid (Merck, Darmstadt, Germany). Optical density in each well was read at 495nm (Hitachi Spectophotometer, Tokyo, Japan) against blank wells and control wells without added serum. Concentrations of TNF $\alpha$ were estimated by the reduction of the optical density of control wells by unknown samples applying a standard curve generated by standard concentrations. All determinations were performed in quadruplicate. The inter-day variation of the assay was $13.75 \%$.

\section{Statistical Analysis}

Results were expressed by their means \pm standard errors (SE). Comparisons were performed by ANOVA; values were adjusted according to Bonferroni to avoid any random correlation. Survival was estimated by Kaplan-Meier analysis. Any value of $\mathrm{p}$ equal to or below 0.05 was considered as significant. Correlations between parameters were performed according to Spearman's rank of order.

\section{RESULTS}

Over the 21-day follow-up, death occurred in 22 animals (mortality $91.67 \%$ ); mean \pm SE survival was $5.23 \pm 1.06$ days.

No macroscopic skin alterations were observed in any animal during necropsy. Histopathological findings of the dermis drawn on necropsy from animals challenged by multidrug-resistant $P$. aeruginosa isolate was more significant than the epidermal alterations. Mean \pm SE total histology score of dermis was $4.41 \pm 0.62$, while mean \pm SE total histology score of epidermis was $0.08 \pm 0.08$ ( $\mathrm{p}<0.0001)$. Main histological findings of dermis after bacterial challenge were inflammation and edema; mean values \pm SE were 0.37 \pm 0.13 and $0.83 \pm 0.15$ respectively. Statistically lower histology scores were found for findings of hair follicles after bacterial challenge compared to edema of the dermis ( $\mathrm{p}$ : 0.047 ) and pathologic lesions of vessels (p: 0.007) respectively. Moreover, a significant positive correlation was found between edema of the dermis and survival time of rabbits.

Both rabbits that survived had edema of the dermis graded as 1 ; mean \pm SE for those who died was $0.81 \pm 0.17$. Indicative pathologic lesions of vessels and hair follicles and inflammation in the dermis of rabbits challenged by the test isolate are shown in Fig. (1).

Mean $\pm \mathrm{SE}$ TNF $\alpha$ at 24 hours was $47.78 \pm 27.63 \mathrm{pg} / \mathrm{ml}$; respective values at 48 hours were $120.30 \pm 62.18 \mathrm{pg} / \mathrm{ml}$. Negative correlations were found between serum concentrations of TNF $\alpha$ at 24 hours and total histology score of dermis $\left(\mathrm{r}_{\mathrm{s}}\right.$ : -0.600 , p: 0.049$)$, as well as between serum concentrations of TNF $\alpha$ at 24 hours and histology score of follicles $\left(r_{s}\right.$ : -0.733 , p: 0.010). No correlation was found between serum $\mathrm{TNF} \alpha$ at 24 hours and survival $\left(\mathrm{r}_{\mathrm{s}}:-0.030\right.$, pNS) as well as between serum TNF $\alpha$ at 48 hours and survival $\left(r_{s}:-0.123\right.$, pNS).

\section{DISCUSSION}

$P$. aeruginosa infection can produce a wide array of manifestations involving skin and soft tissue. Cutaneous infections caused by $P$. aeruginosa can cover a clinical wide spectrum of pathologic entities, ranging from localized skin lesions to extensive skin involvement indicative of potentially life-threatening sepsis in immunocompromised and severely ill patients [7-9]. P. aeruginosa constitutes a common cause of nosocomial sepsis in Intensive Care Units [10]. Pathogens are often multidrug-resistant; their incidence is continuously increasing [11]. The purpose of the present study was to investigate the presence of alterations of the epidermis, dermis and subcutaneous tissue in the setting of experimental sepsis and to correlate these findings with the 
a)

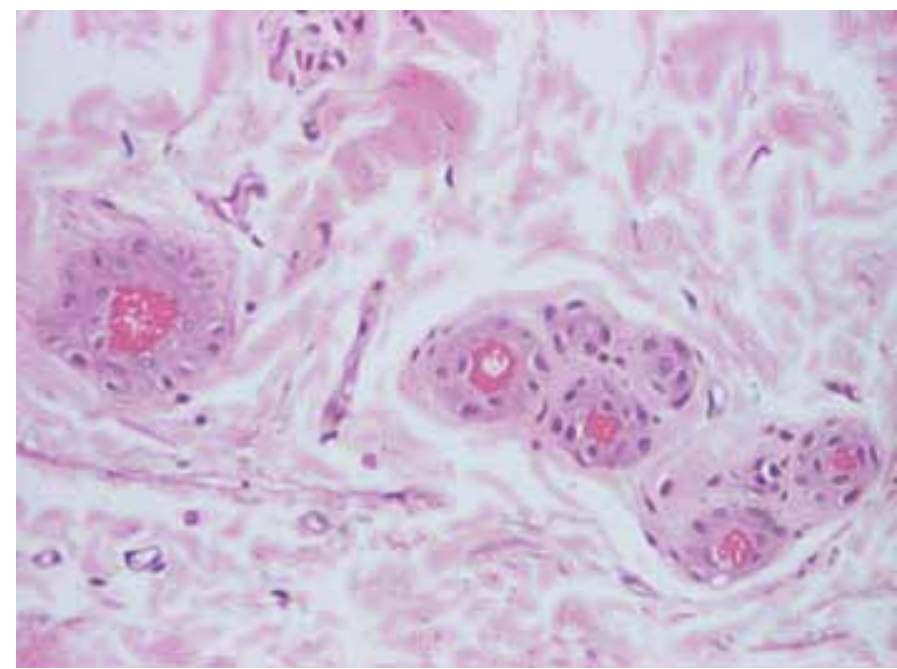

c)

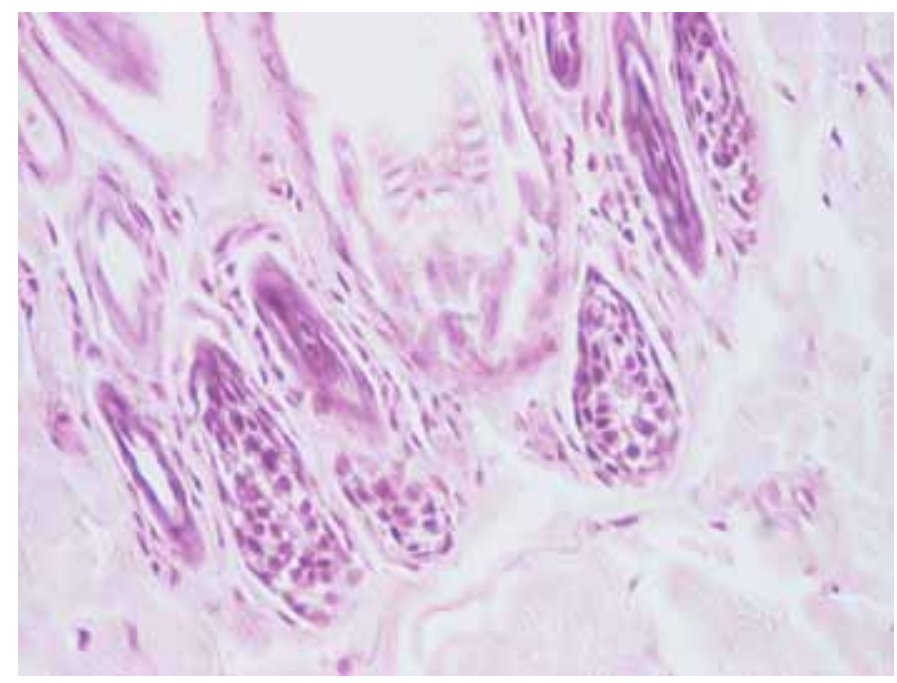

b)

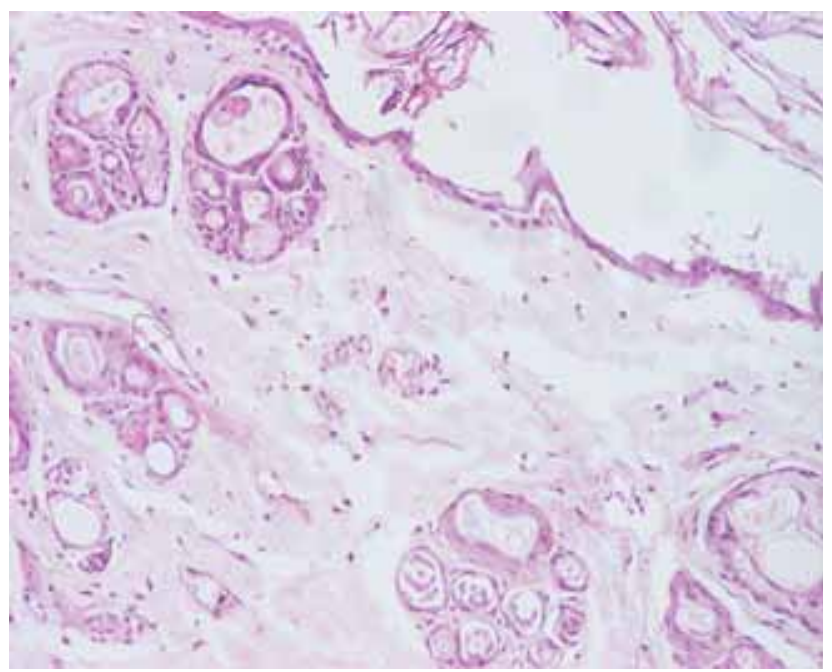

d)

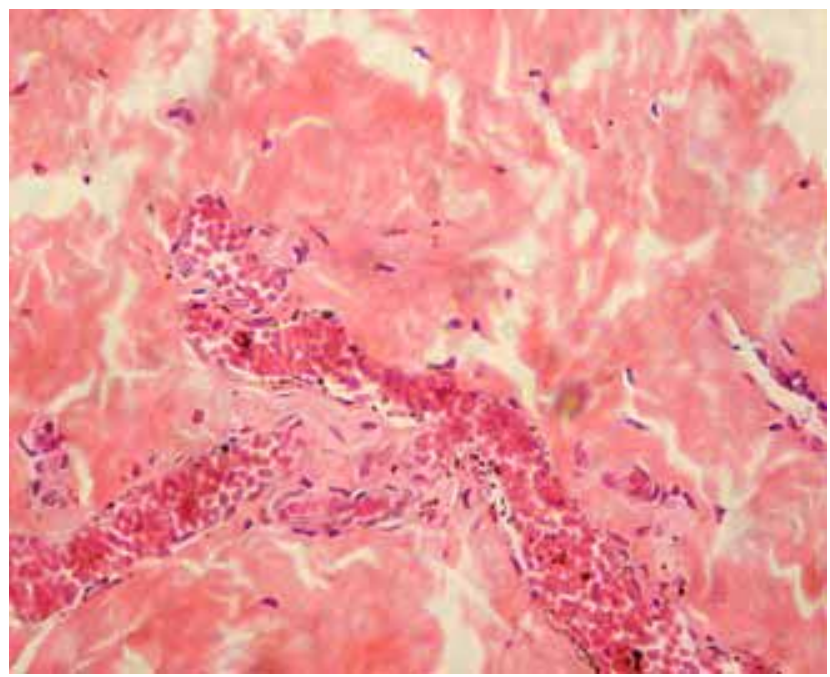

e)

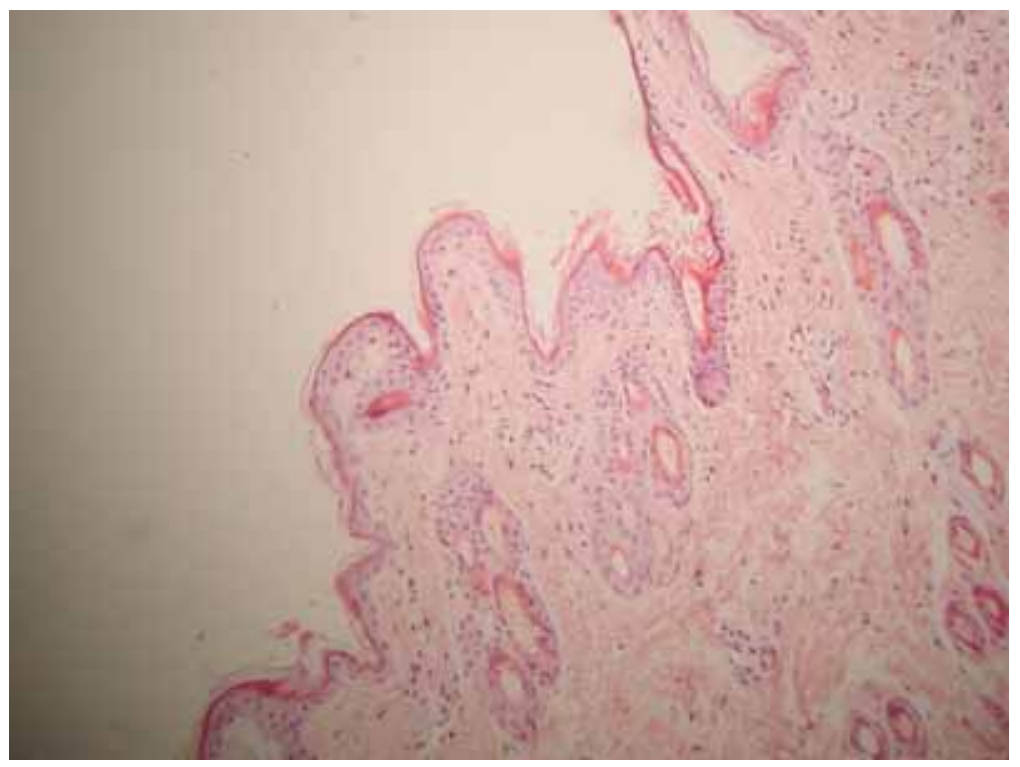

Fig. (1). Histologic findings in skin of rabbits challenged by multidrug-resistant Pseudomonas aeruginosa at death: a) Vessels with thickening of endothelium in the dermis; b) Inflammation and mild edema of the dermis; c) Presence of dyskeratotic cells of hair follicles in the dermis; d) Vessel with the presence of thrombi in the dermis; e) Normal skin histology from a healthy rabbit. 
overall survival after bacterial challenge by multidrugresistant $P$. aeruginosa isolate. Therefore, it might be possible to know if a punch biopsy of the skin constitutes a significant predictive marker of sepsis outcome.

The applied model of sepsis was lethal, as shown by the almost absolute mortality rates of animals challenged by the test isolate. The histological findings of skin after bacterial challenge were inflammation and edema of the dermis, thickening of vascular endothelium and infiltration of the vessel wall and lumen by polymorphonuclear leukocytes, extravasation of red blood cells, presence of thrombi in vessels, presence of acantholytic-like cells within the wall of the hair follicles and dyskeratotic cells (Fig. 1). Animals had more pathological findings in the dermis than those in the epidermis. Edema of the dermis and pathologic lesions of vessels were accompanied by significant pathological findings of hair follicles. Prolongation of survival was accompanied by significant pathological findings in the dermis and particularly by intense edema.

There is no other study showing correlation between edema of the dermis and the time interval to death. A previous study with smaller number of rabbits revealed that systemic infection by multidrug-resistant $P$. aeruginosa was associated with prolonged survival and generated a greater inflammatory response in the dermis and subcutaneous tissue compared to susceptible $P$. aeruginosa [2]. There is another study in animal models which suggests that the skin lesions in Pseudomonas sepsis are initiated at the capillary level, with transmural centripetal arterial or venous infiltration rather than direct hematogenous initimal invasion. Tissue necrosis and hemorrhage would then be the result of bacterial injury and toxin release rather than of vascular obstruction [12].

There have been some reports on the histological findings of cutaneous lesions by punch biopsy in sepsis by $P$. aeruginosa, but there is no correlation of those with the evolution of sepsis after bacterial challenge or with the survival time. These findings were characterized by a necrotizing and hemorrhagic vasculitis of venules and arterioles without intimal damage [13], occasional thrombosis of dermal vessels, scarce inflammatory infiltrate [14], extravasation of red blood cells [15], and in some cases, mild necrosis of epidermis and moderate infarction of dermis [16]. Although, the histological findings of systemic infection by $P$. aeruginosa in the present study were similar to those reported by casereports in humans, no study exists on the pathological alterations of the skin of the non-immunocompromised human host with sepsis by multidrug-resistant isolates. Moreover, intense edema of the dermis was described as a salient feature of sepsis by $P$. aeruginosa which was not described before. Although the presented results may propose that a punch skin biopsy could be helpful diagnostically regarding the severity of sepsis and progression to death, no data are available whether similar histological alterations may supervene in sepsis induced by other Gram-negative pathogens.

Negative correlation was detected between serum levels of TNF $\alpha$ and the extent of histological findings. This might be consistent with previous observations of our study group revealing lower concentrations of TNF $\alpha$ for animals with prolonged survival in experimental sepsis [17], as well as with the assumption that prolonged survival is mandatory for skin alterations to emerge.

\section{CONCLUSION}

The presented experimental model revealed that systemic infection by multidrug-resistant $P$. aeruginosa associated with significant edema of the dermis was accompanied by considerable prolongation of survival; punch skin biopsy might be a significant predictive factor of sepsis outcome. Therefore, further studies must be conducted to clarify the clinical relevance of these findings.

\section{ABBREVIATIONS}

$\mathrm{TNF} \alpha=$ Tumour necrosis factor-alpha

\section{ACKNOWLEDGEMENT}

The study was supported by an unrestricted educational grant by ABBOTT Laboratories, Chicago, USA.

\section{Authors' Contribution}

VT participated in histology studies, revised the manuscript and approved the final version to be submitted.

HT participated in writing and revising the manuscript and approved the final version to be submitted.

TT participated in estimation of $\mathrm{TNF} \alpha$, revised the manuscript and approved the final version to be submitted.

AS participated in animal studies, revised the manuscript and approved the final version to be submitted.

MR participated in animal studies, revised the manuscript and approved the final version to be submitted.

EJGB participated in study design and statistical analysis, in writing and revising the manuscript and approved the final version to be submitted.

NS participated in study design, drafted the manuscript and approved the final version to be submitted.

\section{REFERENCES}

[1] Rangel Frausto MS. The epidemiology of bacterial sepsis. Infect Dis Clin Nor Amer 1999; 13: 299-311.

[2] Petropoulou H, Giamarellos-Bourboulis EJ, Kavatzas N, et al. Early cutaneous alterations in experimental sepsis by Pseudomonas aeruginosa. Dermatology 2004; 209: 111-6.

[3] Giamarellos-Bourboulis EJ, Adamis T, Laoutaris G, et al. Immunomodulatory clarithromycin treatment of experimental sepsis and acute pyelonephritis caused by multidrug-resistant Pseudomonas aeruginosa. Antimicrob Agents Chemother 2004; 48: 93-9.

[4] Clinical and Laboratory Standards Institute: Performance standards for antimicrobial susceptibility testing. $15^{\text {th }}$ International supplement Wayne, Pennsylvania; 2005.

[5] Berry V, Page R, Satterfield J, et al. Comparative efficacy of gemifloxacin in experimental models of pyelonephritis and wound infection. J Antimicrob Chemother 2000; 45 Suppl. 1: 87-93.

[6] Khalil A, Tullus K, Bartfai T, et al. Renal cytokine responses in acute Escherichia coli pyelonephritis in IL-6-deficient mice. Clin Exp Immunol 2000; 122: 200-6.

[7] Sangeorzan JA, Bradley JF, Kauffman CA. Cutaneous manifestations of Pseudomonas infection in the acquired immunodeficiency syndrome. Arch Dermatol 1990; 126: 832-3.

[8] Rolston KV, Bodey GP. Pseudomonas aeruginosa infection in cancer patients. Cancer Invest 1999; 10: 43-59.

[9] Silvestre Jf, Betlloch MI. Cutaneous manifestations due to Pseudomonas infection. Int J Dermatol 1999; 38: 419-31. 
[10] Tacconelli E, Tumbarello M, Bertagnollio S, et al. Multidrugresistant Pseudomonas aeruginosa blood-stream infection: analysis of trends in prevalence and epidemiology. Emerg Infect Dis 2002; 8: $220-1$.

[11] Gales AC, Jones RN, Turnidge J, et al. Characterization of Pseudomonas aeruginosa isolates: Occurrence rates, antimicrobial susceptibility patterns and molecular typing in the global SENTRY antimicrobial surveillance program, 1997-99. Clin Infect Dis 2001; 32: S146-55.

[12] Teplitz C. Pathogenesis of Pseudomonas vasculitis in septic lesions. Arch Pathol 1965; 80: 297-307.

[13] Dorff GJ, Geimer NF, Rosenthal DR, et al. Pseudomonas septicemia. Illustrated evolution of its skin lesion. Arch Intern Med 1971; 128: $591-5$.
[14]

Greene SL, Su WP, Muller SA. Ecthyma gangrenosum: report of clinical, histopathologic and bacteriologic aspects of eight cases. J Am Acad Dermatol 1984; 11: 781-7.

[15] Schlossberg D. Multiple erythematous nodules as a manifestation of Pseudomonas aeruginosa septicemia. Arch Dermatol 1980; 116: 446-7.

[16] Greene SL, Su WPD, Muller SA. Pseudomonas aeruginosa infections of the skin. Am Phys Pract 1984; 29: 193-200.

[17] Giamarellos-Bourboulis EJ, Koussoulas V, Panagou C, et al. Experimental sepsis using Pseudomonas aeruginosa: the significance of multidrug-resistance. Int J Antimicrob Agents 2004; 24: 357-61. 